\title{
Islamofobia queerizada y resistencias musulmanas queer en tiempos de homonacionalismo
}

\section{Queered Islamophobia and Queer Muslim forms of Resistance in times of Homonationalism}

\section{Daniel Ahmed FERNÁNDEZ GARCÍA ${ }^{1}$}

Dptos. Antropología Social y Estudios Árabes e Islámicos

y Estudios Orientales, Universidad Autónoma de Madrid

danielahmedsaid@gmail.com

Recibido 16/4/2018. Revisado y aprobado para publicación 15/6/2018

Para citar este artículo: Daniel Ahmed FERNÁNDEZ GARCÍA (2018), "Islamofobia queerizada y resistencias musulmanas queer en tiempos de homonacionalismo" en Revista de Estudios Internacionales Mediterráneos, 24, 7188.

Para acceder a este artículo: https://doi.org/10.15366/reim2018.24.005

\section{Resumen}

El presente artículo analiza el homonacionalismo desde el cuestionamiento a los discursos culturalistas dicotómicos que dicho fenómeno plantea. En primer lugar, se describen las vinculaciones históricas entre diversidad sexual y de género, racismo y neoliberalismo. A continuación, se aborda la emergencia de un proceso de queerización de la islamofobia nutrido por la instrumentalización neoliberal de los sujetos queer para promover la representación LGBTQ+fóbica del islam. Acto seguido, se evidencia cómo la relación entre las políticas LGBTQ+ transnacionales y la islamofobia queerizada ha provocado un rechazo hacia lo LGBTQ+ en el marco de los Estados arabo-islámicos postcoloniales. Finalmente, se ahonda en el análisis de las identidades musulmanas queer contemporáneas poniendo el foco en sus formas diferenciadas de resistencia frente a los discursos de oposición entre islam y diversidad sexo-genérica, tanto los provenientes de interpretaciones tradicionalistas del islam, como los islamófobos propios del homonacionalismo.

Palabras clave: islamofobia, neoliberalismo, homonacionalismo, queer, resistencia.

\footnotetext{
${ }^{1}$ Contratado predoctoral en la Universidad Autónoma de Madrid en calidad de beneficiario de la ayuda FPI MICINN. Este artículo es resultado de la participación en el proyecto de Excelencia de I+D+I del MICINN "Participación política, islam y transnacionalidad en el mundo árabo-islámico y en contexto migratorio" (CSO2014-52998-C3-1-P).
} 


\begin{abstract}
This article examines homonationalism through the questioning of the dichotomic culturalist discourses that such phenomenon poses. Firstly, it describes the historical connections between gender and sexual diversity, racism and neoliberalism. Next, it addresses the emergence of a process of queerization of Islamophobia, nourished by the neoliberal instrumentalization of queer subjects with the objective of promoting an LGBTQ+phobic depiction of Islam. Thereafter, it calls attention to how the combination of transnational LGBTQ+ politics and queered Islamophobia caused a rejection towards the LGBTQ+ within the context of Arab-Islamic postcolonial states. Finally, it delves into the analysis of contemporary queer Muslim identities, focusing on their differentiated forms of resistance in the face of the discourses that oppose Islam and gender and sexual diversity, both of those coming from traditionalist interpretations of Islam, and those coming from homonationalism.
\end{abstract}

Keywords: Islamophobia, neoliberalism, homonationalism, queer, resistance.

\title{
Introducción
}

El 29 de agosto de 2017, medio centenar de jóvenes saharauis iniciaban una huelga de hambre como protesta ante la denegación de asilo político por parte de las autoridades españolas competentes, tras varias semanas retenidos en el Aeropuerto Madrid-Barajas bajo la custodia del Cuerpo Nacional de Policía y los vigilantes de seguridad privada del aeropuerto ${ }^{2}$. Tres días más tarde, el 1 de septiembre, se publicaba un comunicado ${ }^{3}$ en el que una decena de entidades asociaciones LGBTQ+, colectivos policiales y sindicatos del Ministerio de Interior entre otras ${ }^{4}-$ mostraban su apoyo a la denegación de asilo y la aplicación del protocolo de rechazo y retorno al país de procedencia a dos de los jóvenes saharauis a causa de un "incidente" en el que éstos presuntamente habían proferido insultos homófobos al traductor del Cuerpo Nacional de Policía encargado de asistirles en el proceso. Como consecuencia de esta decisión, el 4 de septiembre se difundía otro comunicado, esta vez en apoyo a los saharauis expulsados, firmado por más de 40 entidades de muy diversa índole -asociaciones LGBTQ+, organizaciones antirracistas y agrupaciones musulmanas de lucha contra la islamofobia- y por más de 200 personalidades pertenecientes al activismo y a la academia ${ }^{5}$.

Publicado en el blog Orgullos Críticos do Sul bajo el lema "no en nuestro nombre", las entidades y personalidades firmantes denunciaban una medida que consideraban a todas luces desproporcional y que, según su punto de vista, vulneraba el artículo 33 de la Convención de los Refugiados de las Naciones Unidas de 1951 de Prohibición de Expulsión y de Devolución, en la que se recoge de manera excepcional la expulsión de una persona solicitante de asilo siempre y

\footnotetext{
2 "Medio centenar de saharauis en huelga de hambre en Barajas para exigir asilo", Público, 29 de agosto de 2017, disponible en http://www.publico.es/sociedad/medio-centenar-saharauis-huelga-hambre-barajas-exigirasilo.html [consulta: 25 de marzo de 2018].

3 "Incidente de inadmisión de la solicitud de asilo en el aeropuerto de Barajas de dos personas por su comportamiento presuntamente agresivo y homofóbico", comunicado de FSC-CCOO, 1 de septiembre de 2017, disponible en http://www.fsc.ccoo.es/6ba29dac339d53c318b5d7df65ba0c27000050.pdf [consulta: 25 de marzo de 2018].

${ }^{4}$ Para acceder al listado completo de entidades firmantes véase el enlace provisto en la nota anterior.

5 "No en nuestro nombre", comunicado de Orgullos Críticos do Sul, 4 de septiembre de 2017, disponible en https://orgulloscriticos.wordpress.com/2017/09/04/no-en-nuestro-nombre/ [consulta: 25 de marzo de 2018].
} 
cuando ésta "sea considerada, por razones fundadas, como un peligro para la seguridad del país donde se encuentra, o que, habiendo sido objeto de una condena definitiva por un delito particularmente grave, constituya una amenaza para la comunidad de tal país" ${ }^{\prime}$. El texto aludía, además, de manera explícita, al "auge del homonacionalismo islamófobo reflejado en este incidente" y a la creciente instrumentalización de la lucha contra la LGBTQ+fobia como cortina de humo para ocultar intereses xenófobos y racistas. Citando el propio comunicado, "si el objetivo fuera el de expulsar a la homofobia del territorio nacional se nos ocurren ejemplos mucho más graves por los que empezar".

Este "incidente" evidencia la emergencia y la toma de conciencia por parte de ciertos sectores del activismo y la academia en el contexto estatal español y portugués, de un "tipo" específico de islamofobia - denominada aquí "islamofobia queerizada" - vinculada a lo que la académica estadounidense Jasbir Puar definió hace una década como homonacionalismo. Según la autora, el homonacionalismo no es un simple sinónimo de "racismo gay", sino una faceta de la modernidad y un cambio histórico marcado por una reorientación de la relación entre los Estados-nación, el neoliberalismo y la sexualidad en relación a las políticas contemporáneas de securitización, terrorismo y patriotismo (Puar, 2007, 2013). Esta convergencia histórica entre prácticas estatales, políticas LGBTQ+ transnacionales e islamofobia se sustenta en un discurso culturalista que enfrenta dos pretendidas realidades: un bloque occidental, moderno, secular y LGBTQ+friendly, y otro, orientalizado, musulmán, anacrónico y LGBTQ+fóbico. Sucesos como la decisión del gobierno canadiense de conceder asilo de manera exclusiva a aquellos refugiados sirios que pudieran probar su homosexualidad ${ }^{7}$, la demonización del islam que siguió a la masacre LGBTQ+fóbica de Orlando $^{8}$, la instrumentalización islamófoba de la "purga gay" en Chechenia ${ }^{9}$ o el propuesto "incidente", constituyen sólo algunas de las más recientes y llamativas manifestaciones de este fenómeno global.

Ante la consolidación de este tipo de situaciones, el presente artículo persigue un objetivo doble: en primer lugar, desentrañar el fenómeno del homonacionalismo desde el cuestionamiento a los discursos culturalistas dicotómicos que plantea; en segundo lugar, visibilizar las resistencias musulmanas queer frente a tales discursos. En la primera parte del artículo, comienzo describiendo las vinculaciones históricas entre diversidad sexual y de género, racismo y neoliberalismo. A continuación, analizo la instrumentalización de los sujetos queer mediante políticas neoliberales con el fin de promover la representación LGBTQ+fóbica del islam. Finalmente, describo la relación entre las políticas LGBTQ+ transnacionales y la islamofobia queerizada en el proceso colonial de homosexualización de los sujetos queer arabo-musulmanes,

6 "Convención sobre el Estatuto de los Refugiados de 1951", disponible en http://www.ohchr.org/SP/Professionallnterest/Pages/StatusOfRefugees.aspx [consulta: 25 de marzo de 2018]. 7 "Determining gay refugees a difficult task, but risk of bogus claimants is 'exaggerated,' experts say", National Post, 24 de noviembre de 2015, disponible en http://nationalpost.com/news/canada/determining-gay-refugees-a-difficulttask-but-risk-of-bogus-claimants-exaggerated-experts-say [consulta: 26 de marzo de 2018].

8 "Stop Exploiting LGBT Issues to Demonize Islam and Justify Anti-Muslim Policies", The intercept, 13 de junio de 2016 , disponible en https://theintercept.com/2016/06/13/stop-exploiting-lgbt-issues-to-demonize-islam-and-justify-antimuslim-policies/ [consulta: 26 de marzo de 2018].

9 "'Gay Lives Matter' and the Future of Islam", The American Conservative, 24 de mayo de 2017, disponible en http://www.theamericanconservative.com/articles/gay-lives-matter-and-the-future-of-islam/ [consulta: 26 de marzo de 2018].

REIM № 24 (junio 2018)

ISSN: $1887-4460$ 
que ha provocado un "rechazo" hacia lo LGBTQ+ dentro de los Estados arabo-islámicos postcoloniales. En la segunda parte, comienzo señalando cuáles han sido las aproximaciones académicas al estudio de las identidades musulmanas queer contemporáneas. Acto seguido, abordo el análisis de dichas identidades atendiendo a una distinción geográfica y sociopolítica países donde el islam es la tradición ${ }^{10}$ mayoritaria y "Occidente"11 - que permite distinguir dos formas diferenciadas de resistencia frente a los discursos de oposición entre islam y diversidad sexo-genérica. Dichas formas de resistencia se materializan, a su vez, en dos movimientos específicos denominados en el marco de este artículo "movimiento de defensa de los derechos LGBTQ+ en países de mayoría musulmana" y "movimiento transnacional de activismo musulmán queer". En la tercera y última parte, presento algunas conclusiones que sirven de cierre del artículo.

Con tal fin, propongo lo queer en tanto que marco teórico y metodológico a través del cual abordar algunas cuestiones como las que ocupan este artículo -islamofobia, neoliberalismo y políticas internacionales de lucha contra el terrorismo entre otras - reservadas tradicionalmente, al menos en el contexto español, a disciplinas como la antropología, la sociología o las ciencias políticas. El presente artículo se enmarca dentro de una investigación de mayor alcance iniciada en octubre de 2014 y aún inconclusa, motivo por el cual se trata fundamentalmente de un texto de discusión bibliográfica, al que hay que sumar una relectura de artefactos culturales relativos a la problemática planteada ${ }^{12}$ y los resultados preliminares obtenidos de una etnografía queer multisituada - deudora de las ideas de Marcus (1995), Halberstam (1998) y Plummer (2005) sobre los dos movimientos de resistencia recién aludidos. Dicha etnografía se ha materializado a través de una doble observación participante. Por un lado, una observación participante online o virtual (Hine, 2000) llevada a cabo en las páginas web y Facebook de las asociaciones que conforman ambos movimientos. Por el otro, una observación participante presencial en formaciones, cursos especializados, seminarios, conferencias o encuentros de carácter internacional organizados exclusivamente por las asociaciones pertenecientes al movimiento transnacional de activismo musulmán queer. Esto es, en Ciudad del Cabo en el seno de The Inner Circle $^{13}$ entre los meses de junio y septiembre de 2015 y durante el desarrollo de $A I R^{14}$ en octubre

\footnotetext{
10 El uso en este artículo de la palabra "tradición" en tanto que sinónimo de "religión" se basa en las ideas de Talal Asad (1986). Para el autor, la religión no constituye ni una estructura social distintiva, ni una colección heterogénea de creencias, costumbres y valores morales, sino una "tradición", es decir, un conjunto de discursos que aluden a la manera de performar determinadas prácticas desde un punto de vista histórico.

11 "Occidente" es definido y empleado a lo largo del artículo sobre las ideas de Asad (1993, 2003), que entiende dicho "marco" como el espacio ideológico y de valores de las democracias neoliberales contemporáneas "pretendidamente" seculares. Para Asad $(1993,2003)$ y $\operatorname{Mahmood}(2005,2016)$ el secularismo constituye, en realidad, una categoría empleada para la legitimación de los Estados-nación modernos mediante una distinción jerárquica entre una esfera pública y otra privada, relegada en exclusiva a la práctica religiosa. Para un análisis comparado de esta cuestión véase Asad, T., Brown, W., Butler, J. y Mahmood, S. (2013).

12 Películas, documentales, literatura, noticias, manifiestos, comunicados, posters, etc. Estos materiales serán referenciados a lo largo del artículo cuando sea pertinente.

${ }^{13}$ Considerada de forma unánime como la primera asociación contemporánea de personas musulmanas queer. Más información disponible en http://theinnercircle.org.za/index.html [consulta: 27 de marzo de 2018].

${ }^{14}$ Acrónimo de Annual International Retreat, un encuentro anual organizado en Ciudad del Cabo por la asociación The Inner Circle desde 2002 hasta la fecha. Es definido en la página web de dicha asociación como "un espacio para personas musulmanas en el que abordar cuestiones relacionadas con el islam y la diversidad sexual (...) y que acoge talleres, seminarios, espacios creativos y de relajación". Disponible en http://theinnercircle.org.za/air.html [consulta: 27 de marzo de 2018].
} 
de 2015 y noviembre de 2016, en Ámsterdam durante los encuentros del EQMN ${ }^{15}$ noviembre de 2016 y noviembre de 2017, en Madrid en la Plataforma del Orgullo Crítico Madrid $2017^{16}$ entre septiembre de 2016 y septiembre de 2017, y en Barcelona en el marco del proyecto Nasij ${ }^{17}$ desde el inicio de la investigación hasta la fecha.

Finalmente, y teniendo en cuenta que la cuestión queer vertebra el contenido del artículo, considero necesaria una aclaración terminológica previa que facilite la lectura a aquellas personas no familiarizadas con esta disciplina. "Diversidad sexual y de género" y "diversidad sexo-genérica" son expresiones sinónimas y se refieren a todo el espectro de orientaciones, prácticas, expresiones e identidades sexuales y de género al margen del sistema binario heteronormativo. Las siglas "LGBTQ+" (lesbianas, gays, bisexuales, personas trans, queer, en cuestionamiento, personas de género no binario, género fluido, tercer género, ad inf.) son categorías sociales e históricas originadas en la "modernidad occidental"18 — aunque en constante definición a nivel global- que hacen referencia a identidades sexo-genéricas específicas. El término anglosajón "queer" aludía originariamente a algo que era extraño o diferente, adquiriendo una connotación peyorativa a finales del s. XIX cuando comenzó a usarse para designar a personas no heterosexuales. Fue resignificado en los 80 desde los activismos LGBT en EE.UU. y Europa con el sentido de identidad positiva. Queer puede referirse a la teoría queer ${ }^{19}$, pero también a los estudios queer 0 al activismo queer. Suele usarse como término paraguas que engloba todas las personas al margen de la heteronorma, en oposición a las identidades LGBTQ+ hegemónicas o, incluso, en oposición a la propia normatividad queer ${ }^{20}$, enfatizándose su carácter de oposición a lo legítimo, a la hegemonía, nunca una cualidad, sino una identidad sin esencia que resiste a los regímenes de lo normal (Halperin, 1997; Warner, 1999) ${ }^{21}$.

\section{Desentrañando el homonacionalismo: entre una islamofobia de género y otra queerizada}

Previamente a que Puar acuñara el concepto de homonacionalismo, Jasmin Zine ya alertaba de las complejas interacciones entre raza, género y religión trazando un paralelismo directo entre las prácticas imperialistas coloniales y la Guerra contra el Terror. La autora señalaba la emergencia de

\footnotetext{
${ }^{15}$ Acrónimo de European Queer Muslim Network, una plataforma europea de activistas musulmanes y musulmanas queer creada en 2015 y coordinada desde 2016 por Maruf, una asociación holandesa "representante de los intereses de las personas musulmanas queer en los Países Bajos y en el extranjero". Más información disponible en http://www.maruf.eu/english.html [consulta: 27 de marzo de 2018]

16 Para acceder a información sobre el origen, objetivos, estrategias y acciones de esta Plataforma véase https://orgullocritico.wordpress.com/ [consulta: 28 de marzo de 2018].

17 Definido en su página de Facebook como un "proyecto concebido como una red física y virtual de resistencia frente a la islamofobia y al homonacionalismo". Disponible en https://www.facebook.com/Nasij-1487428971539744/ [consulta: 28 de marzo de 2018].

18 Defino "modernidad occidental" sobre la base de las ideas de Bryan S. Turner (1990), y los ya nombrados Asad $(1993,2003)$ y Mahmood $(2005,2016)$. Turner entiende la modernidad en relación a la expansión del imperialismo europeo en el s. XVI y al advenimiento del capitalismo racional. Asad Y Mahmood, como vimos, definen "Occidente" como el espacio ideológico de las democracias neoliberales contemporáneas "pretendidamente" seculares.

${ }^{19}$ Nombrada así por primera vez por Teresa de Lauretis en 1990.

20 Peter Drucker dedica su "Warped. Gay Normativity and Queer Capitalism" (2015) de manera específica a esta cuestión.

${ }^{21}$ Para un análisis pormenorizado de dicha terminología véase Barker y Scheele (2017).
} 
una generización de la islamofobia -islamofobia generizada o islamofobia de género (Zine, 2004) - para referirse a las formas individuales, ideológicas y sistémicas de dominación que operaban de manera interseccional (Crenshaw, 1989) en función del género sobre la alteridad musulmana - tanto la femenina como la masculina - en este contexto de "guerra". La dominación de las mujeres musulmanas fue justificada mediante un discurso "civilizador" y de "liberación" de la supuesta opresión patriarcal ejercida por sus homólogos masculinos, que sirvió de legitimización a las intervenciones militares de EE. UU. y sus fuerzas aliadas en Oriente Medio. En el caso de los hombres musulmanes, dicha dominación se llevó a cabo mediante una praxis de humillación sexual que evidenciaba la imbricación entre racismo, misoginia, homofobia, hipermasculinidad y patriotismo, tal y como evidenciaron las grotescas imágenes de la prisión iraquí de Abu Ghraib (Burnham, 2004). Según Zine, estas formas de humillación, lejos de constituir un fenómeno novedoso, estaban fuertemente inspiradas en obras como "The Arab Mind" (1979), del antropólogo Richard Patai, evidenciando el rol del conocimiento orientalista (Said, 1979) a la hora de modelar y ejercer violencia sobre las corporalidades musulmanas (Zine, 2006; Whitaker, 2004).

Jasbir Puar (2007) irá un paso más allá en el análisis de las conexiones entre sexualidad, raza, género, clase y etnicidad en relación a las políticas contemporáneas de la Guerra contra el Terror, al incorporar dos cuestiones ausentes en las teorías de Zine: el neoliberalismo, ligado al concepto de homonormatividad de Lisa Duggan (2002), y la diversidad sexual y de género. Gracias a ello pudo poner de relieve la existencia de otro tipo de corporalidades musulmanas como las LGBTQ+ sobre las que se ejercen otras formas específicas de dominación. Tal y como avancé en la introducción, la autora acuñaba el concepto de homonacionalismo para denunciar un fenómeno histórico contemporáneo en el que están convergiendo diversas prácticas estatales, políticas LGBTQ+ transnacionales y la emergencia de nuevos discursos islamófobos nutridos de la instrumentalización de lo LGBTQ+. Este fenómeno se sustenta en un discurso culturalista, esencialista y homogeneizador que promueve una visión dicotómica del mundo, muy en la línea de la perspectiva académica del "choque de civilizaciones" de Samuel P. Huntington (1996). "Occidente" - moderno, secular y LGBTQ+friendly- y su alteridad - musulmana, anacrónica y LGBTQ+fóbica - son presentados como dos entes monolíticos y antagónicos abocados al enfrentamiento. Según Puar, dicho enfrentamiento se materializa a través de la producción transnacional de dos sujetos antagónicos: "sujetos nacionales queer" y "poblaciones terroristas racializadas musulmanas", tal y como las define la propia autora.

El escenario estadounidense - y el europeo en menor medida - son para Puar el campo de batalla paradigmático de la materialización del enfrentamiento entre ambos sujetos, que define de la siguiente manera. El homonacionalismo promueve una suerte de superioridad identitaria de los "sujetos nacionales queer" respecto de las "poblaciones terroristas racializadas musulmanas". Los primeros pasan a ser considerados sujetos plenos de ciudadanía por parte de los Estados-nación siempre y cuando comulguen y participen de los intereses de los mismos, lo cual se hace a expensas de su desmovilización, despolitización y privatización, así como de su complicidad en la subalternización, discriminación y criminalización de las "poblaciones terroristas racializadas musulmanas". Estas últimas no son consideradas ciudadanas en tanto que encarnan las ideas neoorientalistas que vinculan islam con fundamentalismo, irracionalidad, falta de agencia (Mahmood, 2005), lascivia y/o represión y, especialmente, LGBTQ+fobia. La idea de que las personas musulmanas son más proclives a la LGBTQ+fobia que las que se adscriben al secularismo o a otras tradiciones religiosas como el cristianismo o el judaísmo, responde a la firme creencia de la 
Fernández García, Islamofobia queerizada y resistencias...

incompatibilidad entre islam y diversidad sexo-genérica (Leak y Finken, 2011)22. El homonacionalismo no niega la existencia de personas musulmanas queer, pero entiende que sobre ellas se extiende una suerte de "monstruosidad queer" que sólo puede ser redimida a través de un "secularismo neoliberal queer" que presuntamente sólo se puede materializar en EE. UU y Europa.

El conflicto palestino-israelí, por su parte, constituye para Puar un ejemplo excepcional del enfrentamiento entre ambos sujetos ya que se sitúa - al menos geográficamente - fuera de los escenarios recién señalados. En este caso particular, el Estado de Israel se autoproclama como la única democracia y territorio gay-friendly de Oriente Medio mediante una estrategia de pinkwashing ${ }^{23}$ que le permite producir "sujetos nacionales queer" - dentro y fuera de Israel- que representen, apoyen y legitimen lo que la autora denomina "tecnologías antiterroristas excepcionales" como las políticas genocidas contra el pueblo palestino. La población de Palestina - y por extensión de los países árabes y de mayoría musulmana que le son limítrofes - se convierte de forma automática en agente de opresión queer. Según Puar, conceptualizar Palestina en tanto que territorio LGBTQ+fóbico e Israel en calidad de paraíso gay-friendly, permite al Estado de Israel poner el foco en dicha LGBTQ+fobia, invisibilizando la opresión ejercida por la ocupación israelí sobre la población queer palestina. Asimismo, esta estrategia permite obviar que los privilegios en materia LGBTQ+ que el estado de Israel garantiza son reservados sólo a determinadas personas atendiendo a criterios excluyentes como la clase, la adscripción religiosa o la nacionalidad. Más allá de este conflicto, el término pinkwashing ha proliferado en el ámbito de la academia, el activismo, las redes sociales y los medios de comunicación para referirse a todas las estrategias de lavado de imagen mediante la instrumentalización de lo LGBTQ+.

Según Puar, la aceptación generalizada del discurso dicotómico que sustenta el homonacionalismo - esto es, la presunta incompatibilidad entre islam y diversidad sexo-genérica y la amenaza del islam a los valores de lo que denomina el "reinado queer transnacional" - , ha contribuido a un proceso de intersección entre una "islamofobia gay global" o "islamofobia homonormativa" ${ }^{24}$ y las fuerzas políticas de una derecha gay-friendly que ha comenzado a ver las comunidades LGBTQ+ homonormativas como aliadas naturales (Abraham, 2010). Jin Haritaworn (2008) incluía, además,

\footnotetext{
${ }^{22}$ La aceptación contemporánea generalizada de las premisas en las que se apoya este discurso de incompatibilidad entre islam y diversidad sexo-genérica se debe, en parte, tanto a la escasez de aproximaciones a esta cuestión desde una perspectiva histórica y transcultural (Murray y Roscoe, 1997:3), como a la casi total ausencia de investigaciones de las actitudes hacia dicha diversidad en países no musulmanes (Adamczyk y Pitt, 2009). La problemática es aún más acuciante cuando se trata de abordar la LGBTQ+ fobia como un compendio de factores geográficos, sociopolíticos, económicos y legislativos que van más allá de la cuestión religiosa (Beckers, 2010).

${ }^{23} \mathrm{El}$ término "pinkwashing" fue originalmente acuñado por Breast Cancer Action en 1985 como parte de la campaña "Think Before You Pink" con el objetivo de denunciar aquellas empresas u organizaciones que, con la excusa de apoyar la lucha contra el cáncer de mama mediante el uso del símbolo de un lazo rosa, obtenían beneficios a través de la venta de productos relacionados con dicha enfermedad. Según Sarah Schulman (2012), no será hasta 2010 cuando el colectivo San Francisco QUIT (Queers Undermining Israeli Terrorism) resignifique el término vinculándolo a la instrumentalización israelí de la causa LGBTQ+ con el fin de maquillar su imagen.

${ }^{24}$ A este respecto, Abraham (2010) habla de una "islamofobia queer" o de una "islamofobia hegemónica queer", trazando así un paralelismo entre el concepto de hegemonía y el de homonormatividad de Duggan (2002). Acuño el término "islamofobia queerizada" porque considero que aúna los matices de las propuestas de Puar y Abraham, al tiempo que permite aludir a la confluencia entre una islamofobia de género (Zine, 2004) y otra queerizada, tal y como explico en este mismo párrafo.
} 
en este proceso de intersección, no sólo a las fuerzas políticas de la derecha gay-friendly, sino también a ciertos sectores de la izquierda y de los feminismos. Según la autora, el resultado de este fenómeno ha sido una reformulación de la retórica de la "misión civilizadora" occidental a la que aludía Zine, que ha tenido como consecuencia lo que, en el marco de este artículo, conceptualizo como la confluencia entre una islamofobia de género y otra queerizada, y que explico a continuación. La diversidad sexo-genérica se ha sumado a la igualdad de género en calidad de argumento legitimador de dicha "misión civilizadora", las políticas LGBTQ+ transnacionales se han aliado con las fuerzas de intervención militar $y$, al ya manido argumento de la liberación de las mujeres musulmanas oprimidas por el patriarcado musulmán, hay que sumar ahora la liberación de las víctimas musulmanas queer de la opresión LGBTQ+fóbica pretendidamente ejercida en las sociedades donde el islam es la tradición mayoritaria (Haritaworn, Tauqir y Erdem, 2006; 2008).

En esta misma línea, Joseph Massad llegaba incluso a denunciar en su "Desiring Arabs" (2008) una neocolonización de los sujetos queer arabo-musulmanes a través de lo que el autor denominó "Gay International" y que definía como un conglomerado de fuerzas políticas occidentales organizaciones de derechos humanos, entidades LGBTQ+ de derechas, movimientos feministas $u$ ONGS afincadas en la región MENA (Oriente Medio y Norte de África) - que compartían la reformulada "misión civilizadora" recién aludida. Según Massad, la "Gay International" constituye una manifestación contemporánea de un proyecto colonial occidental de homosexualización (Roscoe, 1997) -iniciado con la expansión de los movimientos de liberación LGBT en EE. UU. y Europa en la década de los $70-$ que ha tenido como consecuencia una "resistencia" hacia lo LGBTQ+ en el seno de los Estados arabo-islámicos postcoloniales. Desde su publicación en 2008, muchos han sido los debates en torno a las afirmaciones de Massad ${ }^{25}$. Más allá de las evidencias que puedan desprenderse del análisis de las legislaciones adoptadas en materia de diversidad sexo-genérica por tales estados ${ }^{26}$, cabe criticar al autor la consideración de "Occidente" y la región MENA como entes monolíticos, la homogeneización de los agentes implicados en la "Gay International", la sobrevaloración de los logros de las políticas LGBTQ+ transnacionales en la esfera global y, especialmente, la negación de la agencia (Mahmood, 2005) de los sujetos queer arabomusulmanes postcoloniales y el fortalecimiento de la consideración de la diversidad sexo-genérica como exclusivamente occidental y, por ende, de la oposición del islam a dicha diversidad.

Esta suerte de "excepcionalismo sexual occidental" constituye para Momin Rahman (2014) la pieza fundamental de un proceso de triangulación que denomina homocolonialismo y que construye sobre la base del concepto de homonormatividad de Duggan (2002), su crítica a la teoría del "Gay International" de Massad (2008) y el homonacionalismo de Puar (2007). Rahman (2015) describe el proceso de la siguiente manera. La consolidación de lo LGBTQ+ como marcador de acceso a la ciudadanía y del grado de modernidad o pinktesting de la alteridad musulmana produce, a su vez, un "rechazo" hacia lo LGBTQ+ en los Estados arabo-islámicos postcoloniales aunque también fuera de ellos $-{ }^{27}$. Que gran parte de ese rechazo se apoye en un tradicionalismo

\footnotetext{
${ }^{25}$ Véase Awwad (2010); Ahmed (2011); Bayban y Najmabadi (2008); Bracke (2012); Habib (2010, 2009, 2007); Landry (2011); Rahman (2014, 2010); Traub (2008).

${ }^{26}$ Para un análisis detallado de la regulación a nivel nacional e internacional de la diversidad sexo-genérica en países de mayoría musulmana, véase ILGA-RIWI Global Attitudes Survey, disponible en http://ilga.org/downloads/ILGA RIWI Minorities Report 2017 Attitudes to sexual and gender minorities.pdf [consulta: 29 de marzo de 2018].

${ }^{27}$ Este "rechazo" alude a la "resistencia" a la que se refería Massad (2008). Rahman (2014) habla de una "antipatía musulmana a la homosexualidad" o incluso de una "homofobia musulmana" que define como parte integral del proceso de triangulación homocolonialista. Abraham (2010) define esta "resistencia" como "homofobia hegemónica
} 
cultural que defiende la incompatibilidad entre islam y diversidad sexo-genérica, reafirma el discurso dicotómico propio del homonacionalismo que sitúa al islam como alteridad anacrónica a "Occidente". Lo cual, al mismo tiempo, acaba reforzando la profusión de una islamofobia queerizada. Para Rahman, la única forma de interrumpir esta triangulación homocolonialista es a través del análisis de las identidades musulmanas queer contemporáneas. Según el autor, dicho ejercicio permite, por un lado, reconocer que la consolidación contemporánea de lo LGBTQ+ como marcador de acceso a la ciudadanía se haya inexorablemente ligado a una agenda anti-musulmana e islamófoba y, por el otro, visibilizar la oposición de estas identidades frente a los discursos culturalistas que oponen islam y diversidad sexo-genérica - tanto los provenientes de las interpretaciones tradicionalistas del islam, como los del homonacionalismo-.

\section{Resistencias musulmanas queer: desafiando los binarismos}

El estudio de las identidades musulmanas queer contemporáneas es, aún a día de hoy, significativamente limitado. No será hasta la década de los 90 , cuando aparezcan las primeras aproximaciones a la diversidad sexo-genérica e islam desde una perspectiva académica ${ }^{28}$ que ha empleado una metodología multidisciplinar con elementos propios de la antropología, el análisis literario, los estudios culturales comparativos o la investigación histórico-teológica. La primera de estas obras fue la colección "Islamic Homosexualities" (1997) de Murray y Roscoe, a la que siguieron otras como las de Wright y Rowson (1997), El-Rouayheb (2005), Habib (2007, 2009, 2010), Babayan y Najmabadi (2008), Kugle (2010, 2014), Jama (2013), Rahman (2014), Hamzic (2016) y Jahangir y Abdullatif (2016). De entre todas ellas, sólo tres abordan de manera significativa la cuestión de las identidades musulmanas queer en la contemporaneidad. "Queer Jihad" (2013) de Jama, presenta una serie de entrevistas realizadas a activistas y líderes religiosos queer relevantes. "Living out Islam" (2014) de Kugle, analiza el trabajo de 15 activistas musulmanes y musulmanas queer que desarrollan su labor en "Occidente"29. "Homosexualities, Muslim cultures and Modernity" (2014) de Rahman, ofrece una aproximación más compleja resultante de la aplicación de un criterio geográfico y sociopolítico - ausente en Jama y Kugleque posibilita dos niveles de análisis que recojo a continuación: identidades musulmanas queer en países donde el islam es la tradición mayoritaria y aquellas situadas en el marco de "Occidente".

Respecto a las identidades musulmanas queer en los países de mayoría musulmana, predomina, siguiendo a Rahman, una tendencia que parece dar más importancia al estudio del pasado homoerótico arabo-islámico que al análisis de las estrategias de activismo de dichas identidades en la contemporaneidad. Según el autor, el clima actual de oposición entre los dos discursos culturalistas que oponen islam y diversidad sexo-genérica, deja literalmente "poco espacio" para que los y las musulmanas queer de estos países puedan desarrollar narrativas alternativas. La

musulmana". He preferido el uso de LGBTQ+fobia para no circunscribir dicha fobia a las personas homosexuales, sino extendiéndola también al resto del espectro LGBTQ+. Asimismo, he rehusado el adjetivo "musulmana" para no inferir una relación causal entre LGBTQ+fobia e islam, evitando así caer en postulados esencialistas y culturalistas como los que pueden desprenderse de las nomenclaturas de Massad, Abraham o Rahman.

${ }^{28}$ Previamente, se habían escrito algunos textos periodísticos y de viajes dedicados a la cuestión. Entre ellos destaca "Sexuality and Eroticism Among Males in Moslem Societies" (1992) de Schmitt y Sofer.

${ }^{29}$ Dichas entrevistas, llevadas a cabo en Sudáfrica, Reino Unido, Países Bajos, EE. UU. y Canadá, permiten al autor identificar 6 grandes modos de activismo: 1. Vinculando la tradición religiosa; 2. Desafiando a la familia y a la comunidad; 3 . Adaptando las políticas religiosas; 4. Adaptando las políticas seculares; 5 . Estableciendo alianzas entre minorías; 6. Caminando hacia la identidad individual. 
mayoría de los esfuerzos han girado, pues, en torno al análisis de las fuentes clásicas del islam —EI Corán, los hádices y la jurisprudencia islámica- y de la literatura homoerótica islámica premoderna ${ }^{30}$, con el objetivo de "rastrear" la existencia de prácticas homosexuales e identidades al margen de la heteronorma ${ }^{31}$ desde el advenimiento del islam en el s. VII hasta la llegada de la colonización europea a comienzos del s. XIX. Samar Habib (2007), principal autora de esta corriente neoesencialista ${ }^{32}$, justifica la necesidad de este enfoque aludiendo a la predominancia en los estudios de género y sexualidad de una epistemología constructivista -influenciada por las teorías expuestas por Michel Foucault en su "Histoire de la sexualité" (1976) ${ }^{33}$ - según la cual algunos autores ${ }^{34}$ han presupuesto la inexistencia de identidades homosexuales más allá de los límites de la "modernidad occidental".

Previamente, Murray y Roscoe (1997), habían señalado cómo dicha epistemología constructivista evoca una suerte de concepción evolucionista de la historia de la homosexualidad al considerar que las personas queer premodernas fueron incapaces de formular sus identidades sobre la base de sus experiencias y prácticas, que nunca crearon redes entre ellas, en definitiva, que los conceptos de identidad y subcultura son invenciones inequívocamente modernas y occidentales. En este sentido, continúan ambos autores, si bien es cierto que no existe ninguna categoría en la historia de los territorios históricos del islam que aglutine de manera inequívoca todos los elementos que conforman las categorías LGBTQ+ contemporáneas, ninguno de estos elementos pertenece en exclusiva a tales categorías, puesto que cada cultura produce formas específicas de deseo sexual y diferentes tipos de identidades (Stein, 1990). En esta misma línea y conectando con los debates acerca de la agencia (Mahmood, 2005) de los sujetos queer árabo-musulmanes postcoloniales a los que se refería Massad, autoras como Evelyn Blackwood concluyen, tras un minucioso trabajo etnográfico sobre lesbianismo en Indonesia, que el hecho de que las categorías LGBTQ+ tengan su origen en la "modernidad occidental", o que hayan sido impuestas por el proceso colonial de homosexualización iniciado en la década de los 70 , no implica que tales categorías no puedan ser reapropiadas y resignificadas por las corporalidades postcoloniales mediante procesos de hibridación (Bhabha, 1994) dando lugar a "identidades locales diferenciadas" contemporáneas (Blackwood, 2005a, 2005b, 2010).

En efecto, el análisis resultante de la observación participante virtual llevada a cabo en las páginas web y Facebook de las asociaciones conformadas por estas "identidades locales diferenciadas"

\footnotetext{
${ }^{30}$ El homoerotismo ha sido considerado por Wright y Rowson (1997) como una tendencia en sí misma dentro de la literatura arabo-islámica premoderna. Algunos de los literatos más estudiados desde esta perspectiva son Ibn Hazm, Abu Nawas, Omar Khayyam, Rumi, Ibn El-Farid o Hafez.

${ }^{31}$ Destaca el análisis de identidades genéricas no normativas de colectivos como las hijras (tercer género) en Asia, las virgjeneshtë (mujeres que adoptan roles de género masculino) en los Balcanes o los mukhannathun (eunucos, hombres afeminados, mujeres trans y personas intersexuales) en la península arábiga. Este último colectivo ya había sido abordado con anterioridad por autoras como Everett Rowson en su "The Effeminates of Early Medina" (1991). Para más información sobre estas cuestiones, véase Kugle (2010); Abdulhadi (2010); Habib (2009, 2007), Babayan y Najmabadi (2008); El-Rouayheb (2005); Murray y Roscoe (1997).

32 Samar Habib es, además, la única autora que se ha centrado en exclusiva en el estudio del lesbianismo en la tradición arabo-islámica. Según Habib, las prácticas homosexuales entre mujeres han sido mayoritariamente ignoradas e indocumentadas a consecuencia de las concepciones patriarcales y falocéntricas de la sexualidad en la jurisprudencia clásica islámica. La autora añade cómo este ámbito de estudio sigue estando predominado por lo que ella misma denomina "epistemología del harén" (Habib, 2009) y que define en tanto que discurso neocolonial que continúa imponiendo sus visiones orientalistas a las mujeres musulmanas.

${ }^{33}$ En esta obra, Foucault afirmaba que la categoría "homosexual" no eran más que una construcción surgida desde el ámbito del psicoanálisis a finales del s. XIX con el objetivo de designar una patología clínica.

${ }^{34}$ Greenberg (1988), Rowson (1991) y Schmitt y Sofer (1992) entre otros.
} 
confirma la existencia de lo que he denominado "movimiento de defensa de los derechos LGBTQ+ en países de mayoría musulmana". Esto es, un movimiento de índole activista ${ }^{35}$, articulado en torno a las identidades sexo-genéricas LGBTQ+, con una clara orientación de incidencia política y social, alejado de los debates teológicos y con un marcado carácter étnico-racial-nacional-regional que se materializa en dos grandes áreas geopolíticas: la región MENA (Medio Oriente y Norte de África) y la región de Asia Central y Sudeste Asiático. Sirva de ejemplo ilustrativo de lo recién expuesto el análisis comparado entre las asociaciones Aswat (Marruecos), Shams y Mawjoudin (ambas en Túnez) y Naz Foundation (Pakistán, India y Sudeste Asiático). Las tres primeras se desarrollan en la región MENA, emplean el francés y el árabe como lenguas vehiculares y todas ellas hacen mención explícita en sus declaraciones de objetivos al fomento de un cambio social con respecto a la percepción de la sexualidad y de la diversidad sexo-genérica, así como a la obtención de un marco legal de protección para la comunidad LGBTQ+ de los países de dicha región. Naz Foundation, por su parte, se desarrolla en la vasta región de Asia Central y Sudeste Asiático, emplea el inglés como lengua común y alude, al igual que las anteriores, a la lucha por los derechos del colectivo LGBTQ+ en los países donde se desarrolla su acción. En ninguno de los casos se hace referencia explícita a la necesidad de conciliación entre orientación sexual e identidad de género e islam o a la legitimación de sus identidades, prácticas o experiencias a través de la tradición musulmana. Lo cual, por oposición, nos lleva a preguntarnos acerca de las identidades musulmanas queer que sí sitúan el islam como elemento vertebrador de sus estrategias de activismo.

Al igual que sucedía con sus homólogas en los países de mayoría musulmana, los discursos culturalistas oposicionales han afectado de manera significativa al modo en que las identidades musulmanas queer occidentales han sido conceptualizadas, hasta el punto de que autores como Abraham (2009) se refieran a ellas como "imposibles" o "inviables". Paradójicamente, esta presunta "imposibilidad" se convertirá en el génesis de lo que he denominado "movimiento transnacional de activismo musulmán queer". La primera de las asociaciones de este movimiento en visibilizarse en la esfera pública transnacional (Fraser, 1990) fue Al-Fitra Foundation -conocida a posteriori como The Inner Circle- en la Sudáfrica post apartheid en 1996, cuando una pequeña comunidad musulmana sufí liderada por el imam Muhsin Hendricks fue consciente de la necesidad de crear una red de apoyo para las personas musulmanas LGBTQ $+{ }^{36}$. Un año más tarde, en 1997, Ilegaría el turno a Al-Fatiha Foundation, fundada a manos de Faisal Alam en Nueva York con el fin de proveer un espacio seguro en el que las personas musulmanas LGBTQ+ pudieran llevar a cabo un proceso de conciliación entre su orientación sexual e identidad de género e islam ${ }^{37}$. Desde

\footnotetext{
${ }^{35}$ Entre las asociaciones más activas destacan Aswat (Marruecos), Mawjoudin y Shams (Túnez), Bedayya y Mesahat (Egipto y Sudán), Al Qaws y Aswat (Palestina), Helem y Meen (Líbano), KAOS GL y Lambdaistanbul Lgbti (Turquía), Iraqueer (Irak), Queer Muslim Project (India), Boys of Bangladesh (Bangladesh), Naz Foundation (Pakistán, India y Sudeste Asiático) o Komunitas Sehati Makassar (Indonesia). Enlaces a cada una de ellas en el apartado de webgrafía [consulta: 1 de abril de 2018]

${ }^{36}$ Dicho hito histórico coincidía con la aprobación de la Constitución de la República de Sudáfrica que vedaba en su Declaración de Derechos (Capítulo 2: Sección 9) la discriminación por orientación sexual y de género entre otros derechos fundamentales. Más información sobre el origen de esta asociación, disponible en la página web de la asociación http://www.theinnercircle.org.za/about-us.html [consulta: 2 de abril de 2018].

37 Texto completo de su "Mission Statement" disponible en http://www.tegenwicht.org/16 imams/al fatiha en.htm [consulta: 2 de abril de 2018].
} 
entonces, el número de asociaciones no ha parado de crecer $^{38}$, especialmente en la década de los $2000^{39}$, evidenciando lo que Habib definió en 2007 como la emergencia de una "yihad queer global".

La conceptualización de esta "yihad queer global" como "movimiento transnacional de activismo musulmán queer" es fruto del análisis derivado de la doble observación participante - virtual y presencial- llevada a cabo en el seno del propio movimiento ${ }^{40}$. Dicho análisis confirma que nos encontramos ante un movimiento diferenciado del "movimiento de defensa de los derechos LGBTQ+ en países de mayoría musulmana" tal y como argumento a continuación. Si bien ambos pueden enmarcarse en el paraguas de los movimientos sociales LGBT (Bernstein, 2002), sólo el desarrollado en el marco de "Occidente" puede ser catalogado como activismo musulmán (Peace, 2015), ya que es el único de los dos movimientos vertebrado en torno al islam -entendido aquí en tanto que tradición religiosa-. Su carácter "queer" y "transnacional" —en oposición al movimiento anterior LGBTQ+ y étnico-racial-nacional-regional- emana de su participación en lo que Blackwood (2008) define como "discursos transnacionales de conocimiento queer", esto es, las formaciones, cursos especializados, seminarios, conferencias o encuentros de carácter internacional señalados con anterioridad ${ }^{41}$. Por último, la doble adjetivación "musulmán-queer" proviene del objetivo primordial del propio movimiento que, según Rahman (2014), gira en torno a la legitimación teológica de identidades, prácticas y experiencias queer a través de los límites del islam. Esta legitimación está siendo llevada a cabo mediante un proceso de "queerización de los textos religiosos" (Yip, 2007; 2008) - muy influenciado por las metodologías de hermenéutica de autoras como Fatima Mernissi (2002) o Amina Wadud (1999) - que está dando como fruto el desarrollo de una epistemología islámica queer.

Algunos de los ejemplos más representativos de este fenómeno son la performatividad (Butler, 1993) de ritos islámicos queerizados como son el salat (oración) libre de segregación sexogenérica, el "imamato" (liderazgo en la oración) queer y, especialmente, la celebración de uniones entre personas del mismo sexo. Kugle, uno de los más destacados eruditos musulmanes contemporáneos, dedica un capítulo completo en su "Homosexuality in Islam" (2010) a la reforma de la ley islámica de cara a acomodar estas uniones dentro de los límites del islam, basándose para ello en las ideas de Kecia Ali (2006) ${ }^{42}$. Paradójicamente, el foco de Kugle en esta "acomodación" lleva al autor a centrarse de manera exclusiva en las resistencias provenientes de lo que denomina "tradición islámica", hasta el punto de afirmar que un movimiento como el que nos ocupa sólo

\footnotetext{
${ }^{38}$ A parte de las ya mencionadas The Inner Circle (Sudáfrica) y Al Fatiha (EE. UU) destacan Muslim Alliance for Sexual \& Gender Diversity (EE. UU.), Salaam Canada (Canadá), Safra Project e Imaan (Reino Unido), HM2F (Francia), Maruf (Países Bajos), Liberal-Islamischer Bund (Alemania), II Grande Colibri (Italia) o Nasij (España). Enlaces a cada una de ellas en el apartado de webgrafía [consulta: 2 de abril de 2018]

${ }^{39}$ Coincidiendo con el impacto que siguió al estreno en 2006 del documental televiso "Gay Muslims" de Cara Laban y, especialmente, "A Jihad for Love" de Parvez Sharma en 2007. Como muestra de los debates que siguieron a estos estrenos véase "Hearts and minds", The Guardian, 6 de diciembre de 2007, disponible en https://www.theguardian.com/film/2007/sep/06/gayrights.religion [consulta: 3 de abril de 2018].

${ }^{40}$ Para una información detallada de esta doble observación participante remítase al apartado de introducción.

${ }^{41}$ Entre los que destacan el ya citado AIR (Anual International Retreat) organizado por The Inner Circle en Sudáfrica y celebrado desde 2002, el Retreat for LGBT Muslims \& Their Partners patrocinado por MASGD (Muslim Alliance for Sexual and Gender Diversity) en EE. UU. y celebrado desde 2011, los encuentros del EQMN (European Queer Muslim Network) organizados por Maruf y celebrados en Ámsterdam desde 2015 o las conferencias CALEM propulsadas por HM2F (Homosexuels Musulmans de France) desde 2010 hasta 2014 en Paris. Enlaces a cada una de estos eventos en el apartado de webgrafía.

42 A la que define como la primera erudita musulmana en trabajar desde una perspectiva teológica "seria" esta cuestión (Kugle, 2010).
} 
puede "florecer" en un marco como el occidental que, en sus propias palabras, se caracteriza por poseer sociedades con fuertes sistemas de protección legal de los derechos individuales, constituciones democráticas y sistemas sociales seculares (Kugle, 2010, 2014). Más allá del esencialismo implícito en tales afirmaciones, la mención al secularismo es especialmente problemática. Asad y Mahmood ${ }^{43}$ ya advirtieron como vimos, de los peligros que conlleva oponer secularismo y religión, o inferir una relación exclusiva y excluyente entre secularismo y "Occidente". Asimismo, Kugle parece olvidar que si bien es cierto que "Occidente" posibilita la emergencia de activismos LGBTQ+ -incluyendo los de índole religiosa- a través de un marco legal de reconocimiento de la libertad religiosa y de protección de las minorías sexo-genéricas, el caso concreto de las identidades musulmanas queer no puede analizarse obviando el clima contemporáneo de islamofobia queerizada.

\section{Conclusiones}

El 19 de julio de 2006 era declarado por iniciativa de dos entidades LGBTQ+ europeas -OutRage! de Gran Bretaña y IDAHO de París - como el Día Internacional de Acción contra la Persecución Homófoba en Irán. Las numerosas manifestaciones a nivel internacional que apoyaron tal declaración no tardaron en provocar declaraciones públicas por parte de activistas musulmanes queer como Faisal Amal -fundador de Al Fatiha en $1997^{44}$ - condenando el llamamiento y preguntándose acerca del interés en presentar Irán como ejemplo paradigmático de LGBTQ+fobia ${ }^{45}$. Resulta paradójico observar cómo estos cuestionamientos previos a la acuñación del propio término homonacionalismo en 2007, sirven ahora para ilustrar algunas de las conclusiones que cierran el presente artículo.

La primera de estas conclusiones es que la hostilidad contemporánea hacia las personas musulmanas a nivel global y la consolidación del islam en el escenario geopolítico en tanto que alteridad anacrónica a la "modernidad occidental", no solo son previas al 11 de septiembre de $2001^{46}$ y al inicio de la consecuente Guerra contra el Terror, sino que, además, no pueden ser entendidas sin atender a los ecos de los "fantasmas" de la Revolución Islámica de Irán en 1979 o de la ocupación israelí de Palestina en 1948, tal y como ya apuntaba Said hace casi 40 años en su "Covering Islam" (1981). No en vano, cabe recordar que la principal estrategia del homonacionalismo, el pinkwashing, alude fundamentalmente a un "lavado de imagen" de las políticas genocidas del estado de Israel contra el pueblo palestino.

La segunda, que la consolidación de lo LGBTQ+ como marcador de acceso a la ciudadanía y del grado de modernidad o pinktesting de la alteridad musulmana se haya vinculada a dos agendas una de racismo anti-musulmán e islamofobia queerizada y otra de rechazo hacia lo LGBTQ+ sustentada en discursos tradicionalistas del islam - que parecen abocadas al enfrentamiento. Este clima de oposición moldea, además, de manera significativa los debates e iniciativas tanto de las

\footnotetext{
${ }^{43}$ Consúltese nota 18.

${ }^{44}$ Como vimos, la primera asociación musulmana queer de EE. UU.

45 Para más información sobre ese "incidente" véase el prólogo de "Terrorist Assemblages" (2007) de Puar.

${ }^{46}$ La tendencia a considerar el 9/11 como fecha paradigmática de la consolidación del islam en la esfera pública transnacional es sustentada, entre otras, por la antropóloga turca Nilüfer Göle (2002).
} 
identidades queer musulmanas en países de mayoría musulmana (obtención de un marco legal de protección para el colectivo LGBTQ+), como de las identidades queer musulmanas en Occidente (legitimización de identidades, prácticas y experiencias queer a través del islam).

En tercer lugar, que las identidades queer musulmanas contemporáneas conforman dos formas diferenciadas de resistencia: un "movimiento de defensa de los derechos LGBTQ+ en países de mayoría musulmana" y un "movimiento transnacional de activismo musulmán queer". En este sentido, si bien es cierto que sus iniciativas frente a los discursos binarios de oposición no implican per se una resistencia, los actos vinculados al estigma de subalternidad (Göle, 2003; Spivak, 1988) resultante de su "musulmaneidad" queer -es decir, en tanto que categoría identitaria política asumida deliberadamente que va más allá de su carácter territorial y religioso (Göle, 2007; Meer 2010) - sí son susceptibles de convertirse en "actos cotidianos de resistencia" (Abu Lughod, 1990).

En cuarto lugar, la necesidad de dar un salto de lo queer al "ensamblaje" (Puar, 2007) con el fin de escapar de las ataduras de las políticas de identidad que privilegian lo LGBTQ+ como culmen de la "modernidad occidental" y ser capaces de reconocer expresiones queer aparentemente reactivas o no resistentes a primera vista, como pueden parecer las del doble movimiento de resistencia frente a los discursos oposicionales. Este salto permite, además, relacionar las ideas de "estigma", "subalternidad", "resistencia" y "ensamblaje". Es decir, entender cómo el estigma vinculado al estado de subalternidad de las identidades queer musulmanas se materializa mediante actos de resistencia cotidianos que pasan desapercibidos para la hegemonía.

Finalmente, cómo las cuestiones que vertebran el marco del homonacionalismo señaladas a lo largo del artículo -racismo, islamofobia, secularismo beligerante, capitalismo militarista, vulneración de los derechos de las minorías étnicas, sexo-genéricas y religiosas-, no sólo no son exclusivas a la cuestión del islam, sino que están vinculadas a la supervivencia del sistema neoliberal. Las políticas de reconocimiento e incorporación de determinados sujetos queer en los Estados-nación son consideradas "medidas de benevolencia" propias de los discursos neoliberales de tolerancia y diversidad cultural. Dichas "medidas" son, además, contingentes en función de parámetros como el privilegio racial, la capacidad de consumo, la normatividad genérica y de parentesco, o la integridad corporal (Puar, 2007, xii).

\section{Bibliografía}

ABDULHADI, Rabab (2010): "Sexualities and the Social Order in Arab and Muslim Communities", en HABIB, Samar (ed.): Islam and Homosexuality, Vols. 1 and 2, Santa Barbara, Praeger.

ABRAHAM, Ibrahim (2010): "Everywhere You Turn You Have to Jump into Another Closet: Hegemony, Hybridity, and Queer Australian Muslims", en HABIB, Samar (ed.): Islam and Homosexuality, Vols. 1 and 2, Santa Barbara, Praeger.

ABRAHAM, Ibrahim (2009): "Out to Get Us: Queer Muslims and the Clash of Sexual Civilization in Australia", Contemporary Islam, 3 (1), pp. 79-97.

https://doi.org/10.1007/s11562-008-0078-3

ABU-LUGHOD, Lila (1990): "The Romance of Resistance: Tracing Transformations of Power Through Bedouin Women", American Ethnologist, 1 (17), pp. 41-55.

https://doi.org/10.1525/ae.1990.17.1.02a00030

ADAMCZYK, Amy y PITT, Cassady (2009): "Shaping attitudes about homosexuality: The role of Religion and Cultural Context", Social Science Research, 38, pp. 338-351.

https://doi.org/10.1016/i.ssresearch.2009.01.002

AHMED, Sara (2011): "Problematic Proximities: Or Why Critiques of Gay Imperialism Matter", 
Feminist Legal Studies, 19, pp. 119-132.

https://doi.org/10.1007/s10691-011-9180-7

ALI, Kecia (2006): Sexual Ethics \& Islam. Feminist Reflections on Qur'an, Hadith, and Jurisprudence, Oxford, Oneworld.

ASAD, Talal; BROWN, Wendy; BUTLER, Judith y MAHMOOD, Saba (2013): Is Critique Secular? Blasphemy, Injury, and Free Speech, California, University of California Press.

ASAD, Talal (2003): Formations of the Secular, Stanford, California, Stanford University Press.

ASAD, Talal (1993): Genealogies of Religion, London, The John Hopkins University Press.

ASAD, Talal (1986): The Idea of Anthropology of Islam, Washington DC, Georgetown University Press.

AWWAD, Julian (2010): "The Postcolonial Predicament of Gay Rights in the Queer Boat Affair", Communication and Critical/Cultural studies, 7, pp. 318-366.

https://doi.org/10.1080/14791420.2010.504598

BABAYAN, Kathryn y NAJMABADI, Afsaneh (2008): (ed.) Islamic Sexualities: Translations across Temporal Geographies of Desire, Cambridge, Harvard University Press.

BARKER, Julia y SCHEELE, Meg-John (2017): Queer: A Graphic History, London, Icon Books.

BECKERS, Tilo (2010): "Islam and the Acceptance of Homosexuality: The Shortage of Socioeconomic Well-Being and Responsive Democracy", en Habib, Samar (ed.): Islam and Homosexuality, Vols. 1 and 2, Santa Barbara, Praeger.

BERNSTEIN, Mary (2002): "Identities and Politics: Toward a Historical Understanding of the Lesbian and Gay Movement", Social Science History, 26 (3), pp. 531-81.

https://doi.org/10.1017/S0145553200013080

BHABHA, Homi (1994): The Location of Culture, London, Routledge.

BLACKWOOD, Evelyn (2008): "Transnational Discourses and Circuits of Queer Knowledge in Indonesia", GLQ: A Journal of Lesbian and Gay Studies, 14 (4), pp. 482-507.

https://doi.org/10.1215/10642684-2008-002

BRACKE, Sarah (2012): "From saving women to saving gays: Rescue Narratives and their dis/continuities", European Journal of Women's Studies, 19, pp. 237-252.

https://doi.org/10.1177/1350506811435032

BUTLER, Judith (1993): Bodies that Matter, London, Routledge.

BURNHAM, Linda (2004): "Sexual Domination in Uniform: an American Value", War Times, 18, pp. 3-4.

CRENSHAW, Kimberle (1989): "Demarginalizing the Intersection of Race and Sex: A Black Feminist Critique of Antidiscrimination Doctrine, Feminist Theory, and Antiracist Politics", The University of Chicago Legal Forum, 140, pp. 139-167.

DE LAURETIS, Teresa (1990): “Queer Theory: Lesbian and Gay Sexualities”, Differences: A Journal of Feminist Cultural Studies, 3, pp. 3-18.

DRUCKER, Peter. (2015): Warped. Gay Normativity and Queer Capitalism, Chicago, Haymarkets Books.

DUGGAN, Lisa (2002): "The New Homonormativity: The Sexual Politics of Neoliberalism", en CASTRONOVO, Russ y NELSON, Dana (eds.) Materializing Democracy: Toward a Revitalized Cultural Politics, Durham y London, Duke University Press.

https://doi.org/10.1215/9780822383901-007

EL-ROUAYHEB, Khaled (2005): Before Homosexuality in the Arab-Islamic World, 1500-1800, Chicago, The University of Chicago Press. 
FOUCAULT, Michel (1976): Histoire de la sexualité I (La Volonté de savoir), Paris, Éditions Gallimard.

FRASER, Nancy (1990): "Rethinking the Public sphere: A contribution to the Critique of Actually Existing Democracy", Social Text, 25 (26), pp. 56-80.

GÖLE, Nilüfer (2007): Interpenetraciones: El islam y Europa, Barcelona, Bellaterra.

GÖLE, Nilüfer (2003): "The Voluntary Adoption of Islamic Stigma Symbols", Social Research: An International Quarterly, 70 (3), pp. 809-828.

GÖLE, Nilüfer (2002): “Close Encounters: Islam, Modernity, and Violence", Understanding September 11, New York, The New York Press, pp. 332-344.

https://doi.org/10.1215/9780822375135-002

GREENBERG, David (1988): The construction of Homosexuality, Chicago, University of Chicago Press.

HABIB, Samar (2010): (ed.) Islam \& Homosexuality, Santa Barbara, Praeger.

HABIB, Samar (2009): Arabo-Islamic Texts on Female Homosexuality (850 - 1780 A.D.), New York, Teneo Press.

HABIB, Samar (2007): Female Homosexuality in the Middle East, New York, Routledge.

HALBERSTAM, Jack (1998): Female Masculinity, Durham, Duke University Press.

HALPERIN, David (1997): Saint Foucault: Towards a Gay Hagiography, Oxford, Oxford University Press.

HAMZIC, Vanja (2016): Sexual and Gender Diversity in the Muslim World. History, Law and Vernacular Knowledge, London, I. B. Taurus \& Co. Ltd.

HARITAWORN, Jin, TAUQUIR, Tamsila y ERDEM, Esra. (2008): “Gay Imperialism: Gender and Sexuality Discourse in the War on Terror", Out of Place: Interrogating Silences in Queerness/Raciality, New York, UK: Raw Nerve Books, pp. 71-95.

HINE, Christine (2000): Virtual Ethnography, London, SAGE Publications.

HUNTINGTON Samuel, (1996): The Clash of Civilizations and the Remaking of World Order, New York, London, Toronto, and Sydney, Simon \& Schuster.

JAHANGIR, Junaid y ABDULLATIF, Hussein (2016): Islamic Law and Muslim Same-Sex, London, Lexington Books.

JAMA, Afdhere (2013): Queer Jihad: LGBT Muslims on Coming Out, Activism, and the Faith, California, Oracle.

KUGLE, Scott (2014): Living Out Islam. Voices of Gay, Lesbian, and Transgender Muslims, New York, New York University Press.

KUGLE, Scott (2010): Homosexuality in Islam. Critical Reflection on Gay, Lesbian, and Transgender Muslims, London, Oneworld.

LANDRY, Donna (2011): “Queer Islam and New Historicism”, Cultural Studies, 25, pp. 147-163.

https://doi.org/10.1080/09502386.2011.535983

LEAK, Gary y FINKEN, Laura (2010): "The Relationship between the Constructs of Religiousness and Prejudice: A Structural Equation Model Analysis", The International Journal for the Psychology of Religion, 21, pp. 43-62. https://doi.org/10.1080/10508619.2011.532448

MAHMOOD, Saba (2016): Religious difference in a secular age, New Jersey, Princeton University Press.

MAHMOOD, Saba (2005): Politics of Piety: The Islamic Revival and the Feminist Subject, New Jersey, Princetown University Press.

MARCUS, E. George (1995): "Ethnography in/of the world system: the emergence of multisited ethnography", Annual Review of Anthropology, 24, pp. 95-117.

https://doi.org/10.1146/annurev.an.24.100195.000523

MASSAD, Joseph (2008): Desiring Arabs. Chicago and London, The University of Chicago Press. 
MEER, Nasar (2010): Citizenship, Identity and the Politics of Multiculturalism: The Rise of Muslim Consciousness, Basingstoke, Palgrave Macmillan.

MERNISSI, Fatima (2002): El harén político: el Profeta y las mujeres, Madrid, Ediciones del Oriente y del Mediterráneo.

MURRAY, Stephen y ROSCOE, Will (1997): Islamic Homosexualities. Culture, History, and Literature, New York, New York University Press.

PATAI, Richard (1979): The Arab Mind, Long Island NY, Hatherleigh Press.

PEACE, Timothy (2015): European Social Movements and Muslim Activism. Another World but with Whom?, Basingstoke, Palgrave Macmillan.

PLUMMER, Ken (2005): "Critical Humanism and Queer Theory: Living with the Tensions", en DENZIN, Norman y LINCOLN, Yvonna (eds.) Handbook of Qualitative Research, London, SAGE, pp. 195-208.

PUAR, Jasbir (2013): "Rethinking Homonationalism", International Journal of Middle East Studies, 45, pp. 336-39. https://doi.org/10.1017/S002074381300007X

PUAR, Jasbir (2007): Terrorist Assemblages: Homonationalism in Queer Time, Durham, Duke University Press.

RAHMAN, Momin (2015): "The Politics of LGBT Muslim Identities", International Relations, 2 de abril, 2015, http://www.e-ir.info/2015/04/02/the-politics-of-lgbt-muslim-identities/

RAHMAN, Momin (2014): Homosexualities, Muslim Cultures and Modernity, London, Palgrave Macmillan.

RAHMAN, Momin (2010): "Queer as Intersectionality: Theorizing Gay Muslim Identities", Sociology, 44, pp. 1-18.

ROWSON, Everett (1991): "The Effeminates of Early Medina", Journal of the American Oriental Society, 111, pp. 671-93.

SAID, Edward (1981): Covering Islam, London, Routledge.

SAID, Edward (1979): Orientalism, New York, Vintage Books.

SCHULMAN, Sara (2012): Israel/Palestine and the Queer International, Durham, Duke University Press.

SCHMITT, Arno y SOFER, Jehoeda (1992): Sexuality and Erotism among Males in Moslem Societies, New York, Harrington Park Press.

SPIVAK, Gayatri (1988). "Can the subaltern speak?", Marxism and the Interpretation of Culture, en NELSON, Cary y GROSSBERG, Lawrence (eds.), Urbana: University of Illinois, pp. 271-313.

STEIN, Edward (1990): The Mismeasure of Desire: The Science, Theory, and Ethics of Sexual Orientation, New York, Routledge.

TRAUB, Valerie (2008): "The Past is a Foreign Country? The Times and Spaces of Islamic Sexuality Studies", en BABAYAN, Kathryn y NAJMABADI, Afsaneh (eds.), Islamic Sexualities: Translations across Temporal Geographies of Desire, Cambridge, Harvard University Press.

TURNER, Bryan (2002): "Sovereignty and Emergency: Political Theology, Islam and America", Theory, Culture and Society, 19, pp. 103-119.

TURNER, Bryan (1990): Theories of Modernity and Postmodernity, London, Sage.

WADUD, Amina (1999): Qur'an and Woman. Rereading the Sacred Text from a Woman's Perspective, Oxford, Oxford University Press.

WHITAKER, Brian (2004): Unspeakable Love: Gay and Lesbian Life in the Middle East, London, Saqi. WARNER, Michael (1999): The Trouble with Normal: Sex, Politics and the Ethics of Queer Life, New York, Free Press. 
WRIGHT, Jennifer y ROWSON, Everett (1997): Homoerotism in Classical Arabic Literature, New York, Columbia University Press.

ZINE, Jasmin (2006): "Between Orientalism and fundamentalism: Muslim women and feminist resistance", Muslim World Journal of Human Rights, 2, pp. 1-26. https://doi.org/10.2202/1554$\underline{4419.1080}$

ZINE, Jasmin (2004): "Staying on the Straight Path: A Critical Ethnography of Islamic Schooling in Ontario". Disertación doctoral no publicada, Departamento de Sociología y Estudios de Equidad en Educación, Universidad de Toronto.

\section{Webgrafía}

Asociaciones del "movimiento de defensa de los derechos LGBTQ+ en países de mayoría musulmana" :

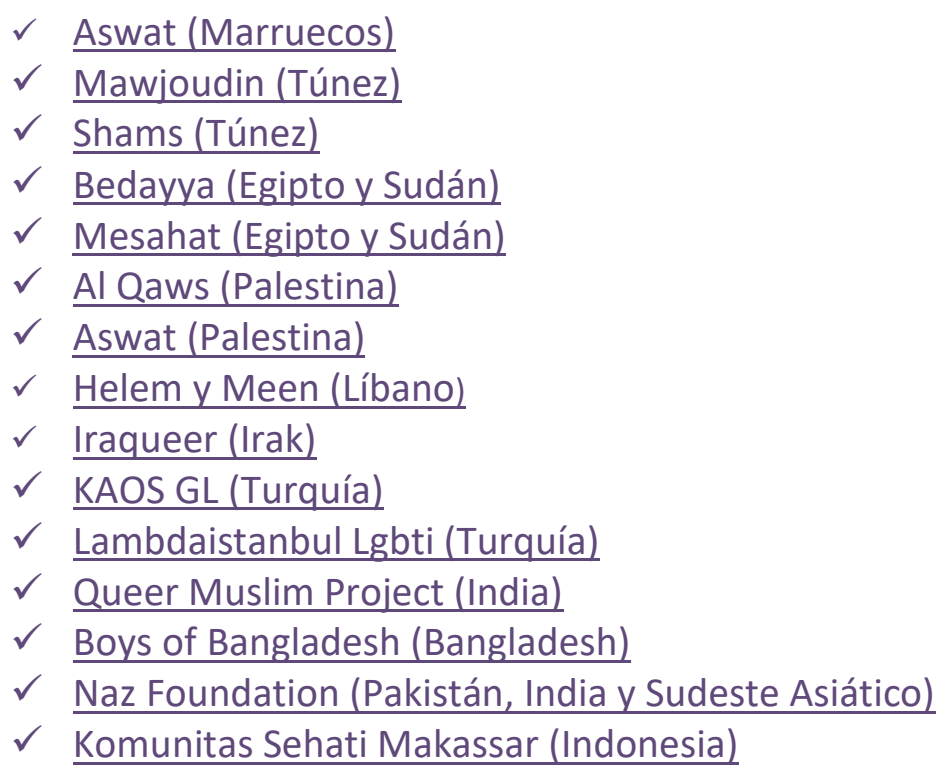

Asociaciones del "movimiento transnacional de activismo musulmán queer":

$\checkmark$ The Inner Circle (Sudáfrica)

$\checkmark$ Al Fatiha (EE. UU)

$\checkmark$ Muslim Alliance for Sexual \& Gender Diversity (EE. UU.)

$\checkmark$ Salaam Canada (Canadá)

$\checkmark$ Safra Project (Reino Unido)

$\checkmark$ Imaan (Reino Unido)

$\checkmark$ HM2F(Francia)

$\checkmark$ Maruf (Países Bajos)

$\checkmark$ Liberal-Islamischer Bund (Alemania)

$\checkmark$ II Grande Colibri (Italia)

$\checkmark$ Nasij (España)

Encuentros de carácter internacional del "movimiento transnacional de activismo musulmán queer":

$\checkmark$ AIR (Anual International Retreat)

$\checkmark$ Retreat for LGBT Muslims \& Their Partners

$\checkmark$ EQMN (European Queer Muslim Network) 